Bundesgesundheitsbl 2020 $63: 415-425$ https://doi.org/10.1007/s00103-020-03110-1 Online publiziert: 18. März 2020

(c) Der/die Autor(en) 2020

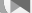

Carsten Oliver Schmidt ${ }^{1}$ Klaus-Peter Günther ${ }^{2}$. Jens Goronzy ${ }^{2}$.

Katinka Albrecht ${ }^{3}$. Jean-François Chenot ${ }^{1}$. Johanna Callhoff ${ }^{3}$. Adrian Richter ${ }^{1}$.

Richard Kasch ${ }^{29}$. Wolfgang Ahrens $s^{5} \cdot$ Heiko Becher $^{6} \cdot$ Klaus Berger $^{7}$.

Hermann Brenner ${ }^{8}$. Beate Fischer ${ }^{14}$. Claus-Werner Franzke ${ }^{22}$.

Wolfgang Hoffmann ${ }^{1} \cdot$ Bernd Holleczek $^{9} \cdot$ Lina Jaeschke ${ }^{19}$. Carsten Jenning ${ }^{1} \cdot$ Karl-

Heinz Jöckel ${ }^{10}$. Rudolf Kaaks ${ }^{8}$. Thomas Keil ${ }^{11,30,31}$ • Alexander Kluttig ${ }^{23}$.

Gérard Krause $^{12}$. Oliver Kuß ${ }^{13}$. Michael Leitzmann ${ }^{14}$. Wolfgang Lieb ${ }^{15}$.

Jakob Linseisen ${ }^{17,18}$. Markus Löffler ${ }^{16,25}$. Claudia Meinke-Franze'

Christa Meisinger ${ }^{17,18} \cdot$ Karin B. Michels $s^{22} \cdot$ Rafael Mikolajczyk ${ }^{23} \cdot$ Nadia Obi $^{6}$.

Annette Peters $^{18}$ • Tobias Pischon ${ }^{19,20,21,31,32}$. Tamara Schikowski ${ }^{24}$. Sabine Schipf ${ }^{1}$. Christof Specker $^{25} \cdot$ Henry Völzke ${ }^{1} \cdot$ Kerstin Wirkner $^{16,26} \cdot$ Angela Zink $^{3,27}$.

Oliver Sander ${ }^{28}$

\title{
Häufigkeiten muskuloskelettaler Symptome und Erkrankungen in der bevölkerungsbezogenen NAKO Gesundheitsstudie
}

logischen Studien keine Entsprechung fanden.

Die muskuloskelettalen Erkrankungen lassen sich in vier zentrale Gruppen einteilen. Dies sind neben Rückenschmerzen die durch degenerative Veränderungen an den Gelenken gekennzeichnete Arthrose, die Osteoporose, deren charakteristisches Merkmal eine Abnahme der Knochendichte ist, sowie chronisch entzündliche Gelenkerkrankungen. $\mathrm{Zu}$ allen gibt es umfassende epidemiologische Anknüpfungspunkte.

Global gesehen sind Rückenschmerzen einer der wichtigsten Gründe für Beeinträchtigungen in der Allgemeinbevölkerung [6, 7]. In einem deutschen Survey gaben ca. $70 \%$ der erwachsenen Teilnehmenden an, im letzten Jahr Rückenschmerzen gehabt zu haben, rund $10 \%$ berichteten von Rückenschmerzen, die mit relevanten Beeinträchtigungen einhergingen [8]. Bei rund $20 \%$ der Bevölkerung traten chronische Rückenschmerzen auf [9]. Hinsichtlich der Häufigkeit des Auftretens ist es wichtig, zwischen Populationsepidemiologie und Versorgungsepidemiologie zu unterscheiden. Die Anzahl der Erwachsenen, die mit ihren Rückenbeschwerden im
Laufe eines Jahres laut AOK zu Patienten werden, ist mit $25 \%$ gegenüber dem Anteil aller Betroffenen deutlich geringer [10]. Gleichzeitig sind Rückenschmerzen für das Gesundheitsversorgungssystem von hoher ökonomischer Bedeutung. Im Gegensatz zur Arthrose sind Rückenschmerzen mit Funktionsbeeinträchtigungen bei der berufstätigen Bevölkerung wesentlich verbreiteter [11]

Während Rückenschmerz primär durch eine subjektiv erlebte Symptomatik definiert ist, die vor allem bei geringen Beschwerden keinen Krankheitswert haben muss, ist die Situation für die anderen muskuloskelettalen Erkrankungen deutlich komplexer. Deren frühe Stadien können symptomfrei verlaufen und müssen den Betroffenen nicht bekannt sein. Überdies können neben Selbstangaben auch klinische oder radiologische Untersuchungen zur Erfassung der Erkrankung verwendet werden, wodurch sich sehr unterschiedliche Häufigkeiten ergeben können [12].

Prävalenzdaten $\mathrm{zu}$ Arthrosen in Deutschland liegen aus verschiedenen Gesundheitssurveys vor, etwa aus der Studie zur Gesundheit Erwachsener in Deutschland (DEGS1) des Robert Koch- 
Instituts von 1997-1999 [13] und einer Befragung von ca. 24.000 Personen im Jahr 2014 im Rahmen der GEDAStudie. Laut GEDA 2012 gaben insgesamt $28 \%$ der Frauen und $20 \%$ der Männer an, dass bei ihnen jemals eine Arthrose ärztlich diagnostiziert wurde [15]. Es werden mehr als 350.000 Hüftund Knieendoprothesen jährlich wegen Gelenkverschleißes in Deutschland implantiert [16]. In DEGS1 wurde auch nach Gelenkschmerzen, unter anderem in den Fingergelenken, gefragt. Diese wurden bei gut $8 \%$ der untersuchten Frauen und $4 \%$ der Männer dokumentiert [17]. Die Prävalenzschätzung in DEGS1 differenziert nicht zwischen den verschiedenen Manifestationen einer Arthrose und beschränkt sich auf die berichtete Diagnose [18].

Bis zum Auftreten der ersten Fraktur ist die Osteoporose eine weitgehend asymptomatische Skeletterkrankung [19]. Daher sind Selbstangaben der Betroffenen nur von eingeschränktem epidemiologischen Wert zur Beurteilung der Verbreitung. Goldstandard zur Erfassung ist die Knochendichtemessung, die aber wegen der Strahlenexposition kaum in bevölkerungsbezogenen Studien eingesetzt werden kann [20]. Betroffen sind vor allem Frauen ab 50 Jahren. Überdies beeinflussen Alter, Komorbiditäten und Medikamente das Osteoporoserisiko [21]. Die Prävalenz in Deutschland wurde anhand von Abrechnungsdaten auf $24 \%$ bei Frauen und auf $6 \%$ bei Männern über 50 Jahren geschätzt [22], rund $50 \%$ hatten eine klinisch manifeste Fraktur. Insgesamt ist von einer Unterschätzung der Häufigkeit von Osteoporose auszugehen.

Entzündlich-rheumatische Erkrankungen sind chronische Autoimmunerkrankungen. Bei unzureichender Krankheitskontrolle führen diese aufgrund der Entzündungsaktivität zu Schmerzen und Funktionsverlust am Bewegungsapparat, aber auch zu Gewebeschäden an zahlreichen Organen [23]. Diese können mit einer erhöhten Morbidität und Mortalität einhergehen. Entzündlichrheumatische Erkrankungen sind seltener als degenerative oder stoffwechselbedingte muskuloskelettale Erkrankungen, jedoch wird geschätzt, dass etwa
$2 \%$ der erwachsenen Bevölkerung an entzündlich-rheumatischen Krankheiten leiden [24]. Die Hände stellen den alleinigen Manifestationsort der Fingergelenksarthrosen und den wesentlichen Manifestationsort der rheumatoiden Arthritis dar. Letztere gilt als die häufigste entzündlich-rheumatische Erkrankung. Es gibt wenige Häufigkeitsangaben aus Deutschland und diese variieren je nach Definition, Stichprobe und Verteilung [25]. Die bevölkerungsbezogene Erfassung gestaltet sich vor allem durch die niedrige Prävalenz problematisch.

Mit der vorliegenden Arbeit werden Häufigkeiten wichtiger muskuloskelettaler Symptome und Erkrankungen auf Basis von Daten der deutschen NAKO Gesundheitsstudie beschrieben. Dabei wird die Verteilung der Symptome und Erkrankungsgruppen nach Geschlecht und Alter betrachtet. Die Häufigkeiten werden vor dem Hintergrund weiterer bevölkerungsbezogener epidemiologischer Studien bewertet. Ein epidemiologisches Alleinstellungsmerkmal ist die Durchführung von klinischen Untersuchungen an den Händen, Knien und Hüften, deren Ergebnisse mit Fokus auf Schwellungen und Schmerzen dargestellt werden.

\section{Methoden}

\section{Studiendesign}

Die Daten wurden in der ersten Hälfte der Basiserhebung der NAKO Gesundheitsstudie von März 2014 bis März 2017 in 18 Studienzentren erhoben. Eingeschlossen wurden über die lokalen Melderegister zufällig ausgewählte Erwachsene, die zum Zeitpunkt der Stichprobenziehung 20 bis 69 Jahre alt waren. Weitere Voraussetzungen für die Teilnahme waren ein Wohnort im Einzugsgebiet des für das Studienzentrum verantwortlichen Einwohnermeldeamtes und ausreichende Deutschkenntnisse [26]. Die Ziehung der Stichprobe erfolgte altersund geschlechtsstratifiziert (50\% Männer/Frauen; dabei jeweils 10-Jahres-Altersgruppen: je $10 \%$ in den zwei unteren, je $26,6 \%$ in den übrigen 10 -JahresAltersgruppen).

Nach Ausschluss von 10 Fällen ohne Altersangaben gingen Daten von
101.779 Studienteilnehmenden aus dem Interview der Basisuntersuchung (Level 1) in die Auswertungen ein. Die klinische Untersuchung der Hand und die Winkelstuhluntersuchung wurden im umfangreicheren Untersuchungsprogramm der sogenannten Level-2Untersuchungen $(N=28.833)$ als ein sogenanntes 1-aus-3-Modul bei 9943 Teilnehmenden durchgeführt. 1-aus-3Modul bedeutet, dass die muskuloskelettale Untersuchung optional durchgeführt werden konnte, wenn in einem Studienzentrum ein besonderes Interesse an diesem Thema bestand.

\section{Erhebungsinstrumente}

\section{Level-1-Basisuntersuchung: Interview}

Das Interview wurde computerunterstützt an allen NAKO-Studienzentren identisch durchgeführt. Erfragt wurde, ob jemals eine der betreffenden muskuloskelettalen Erkrankungen diagnostiziert wurde sowie, bei einer positiven Antwort, deren Behandlung in den vergangenen 12 Monaten. Der Wortlaut der Fragen und Antworten ist in - Tab. 1 aufgeführt. Für Rückenschmerzen gab es keine Frage zur Behandlung in den vergangenen 12 Monaten.

\section{Level-2-Zusatzuntersuchung: klinische Handuntersuchung und Winkelstuhl}

Bei der Handuntersuchung brachte der Untersucher die Hand des sitzenden Teilnehmers in die richtige Position. Die Hand zeigte mit der Handfläche zum Fußboden (Pronation) und war in Höhe der zu untersuchenden Fingergrundgelenke in einem Winkel von etwa $50 \mathrm{Grad}$ im Handgelenk gebeugt und an den Mittelgelenken gestreckt. Jedes Fingergrund- und Mittelgelenk wurde nacheinander mit Zeige- oder Mittelfinger der einen Hand des Untersuchers nach dorsal (oben) gedrückt und mit Zeigefinger und Daumen der anderen Hand palpiert. Wurden beim Abtasten der Gelenke Schwellungen und/oder Druckschmerz erkannt, so wurde dies für das betroffene Gelenk dokumentiert. Eine Gelenkschwellung wurde bei fluktuierender Flüssigkeit, die bei Druck 
Bundesgesundheitsbl 2020 • 63:415-425 https://doi.org/10.1007/s00103-020-03110-1

(c) Der/die Autor(en) 2020

C. O. Schmidt · K.-P. Günther · J. Goronzy · K. Albrecht · J.-F. Chenot · J. Callhoff · A. Richter $\cdot$ R. Kasch · W. Ahrens $\cdot$ H. Becher $\cdot$ K. Berger .

H. Brenner · B. Fischer · C.-W. Franzke · W. Hoffmann · B. Holleczek · L. Jaeschke · C. Jenning · K.-H. Jöckel · R. Kaaks · T. Keil · A. Kluttig · G. Krause · O. Kuß - M. Leitzmann - W. Lieb - J. Linseisen - M. Löffler · C. Meinke-Franze · C. Meisinger · K. B. Michels · R. Mikolajczyk · N. Obi · A. Peters ·

T. Pischon · T. Schikowski $\cdot$ S. Schipf $\cdot$ C. Specker $\cdot$ H. Völzke $\cdot$ K. Wirkner $\cdot$ A. Zink $\cdot$ O. Sander

\section{Häufigkeiten muskuloskelettaler Symptome und Erkrankungen in der bevölkerungsbezogenen NAKO Gesundheitsstudie}

\section{Zusammenfassung}

Hintergrund. Muskuloskelettale Symptome und Erkrankungen sind in der Bevölkerung sehr weit verbreitet und verursachen hohe Kosten in Gesundheitswesen und Volkswirtschaft.

Ziel der Arbeit. Die Häufigkeit muskuloskelettaler Symptome und Erkrankungen aus den Bereichen Rückenschmerzen, Osteoporose, Arthrose und Arthritis wurde auf Basis von Daten der bevölkerungsbasierten NAKO Gesundheitsstudie beschrieben.

Material und Methoden. Die Daten wurden in der ersten Hälfte der NAKOBasiserhebung von März 2014 bis März 2017 bei Erwachsenen im Alter von 20-75 Jahren erhoben. In die Analysen gingen Daten von 101.779 Interviewteilnehmenden sowie von 9370 Teilnehmenden aus zwei klinischen Untersuchungen ein. Mittels der klinischen Handuntersuchung wurden tastbar geschwollene Gelenke sowie auf Druck schmerzhafte Gelenke identifiziert. Die Winkelstuhluntersuchung lieferte Daten zu Ruheschmerzen am Knie- und Hüftgelenk. Häufigkeiten wurden auf die deutsche Standardbevölkerung 2011 gewichtet. Ergebnisse. In den Selbstberichten der Teilnehmenden im Interview wurden am häufigsten jemals diagnostizierte andauernde Rückenschmerzen (22,5\%) und Arthrosen $(20,6 \%)$ angegeben, seltener Osteoporose $(2,9 \%)$ und rheumatoide Arthritis (1,9\%). In der Handuntersuchung wurden bei 6,0\% Schmerzen an mindestens einem Fingergelenk festgestellt. Laut Winkelstuhluntersuchung waren $8,2 \%$ von einem Ruheschmerz in mindestens einem Knie betroffen sowie 5,1\% an der Hüfte. Frauen berichteten insgesamt häufiger muskuloskelettale Erkrankungen und Symptome als Männer, der Anteil Betroffener nahm mit dem Alter deutlich zu.

Diskussion. Die Ergebnisse belegen die große Verbreitung vieler muskuloskelettaler Erkrankungen. Die berichtete Beschwerdelast ist mit Ergebnissen aus früheren bevölkerungsbezogenen Erhebungen vergleichbar.

\section{Schlüsselwörter}

Muskuloskelettale Erkrankungen · Rückenschmerz $\cdot$ Arthrose $\cdot$ Arthritis - Osteoporose . Allgemeinbevölkerung

\section{Frequencies of musculoskeletal symptoms and disorders in the population-based German National Cohort (GNC)}

Abstract

Background. Musculoskeletal diseases and symptoms are very common in the general population. They lead to high healthcare costs and pose a significant burden to the national economy.

Objectives. Based on data from the population-based German National Cohort (GNC), frequencies of musculoskeletal symptoms and diseases are reported, including back pain, osteoporosis, osteoarthritis, and arthritis. Materials and methods. Data were collected from March 2014 to March 2017 in adults aged 20-75 years during the first half of the baseline survey of the GNC. The sample comprised 101,779 interviewed subjects, including 9370 subjects who underwent clinical musculoskeletal examinations.
The interview included questions about specific musculoskeletal disorders. A clinical examination of the hand provided information about palpable swollen joints and pressuresensitive joints. Resting pain of the knees and hips was also assessed by a clinical examination. Frequencies were standardized to the German standard population of the year 2011.

Results. Having ever been diagnosed with recurrent back pain (22.5\%) or osteoarthritis $(20.6 \%)$ were the most common complaints reported in the interview; osteoporosis (2.9\%) and rheumatoid arthritis (1.9\%) were stated more seldom. According to the hand examination, $6.0 \%$ of all participants experienced pain in at least one finger joint.
Resting pain was present in at least one knee among $8.2 \%$ and in at least one hip among $5.1 \%$ of the participants as assessed during the clinical examination. Women were more likely to report musculoskeletal disorders and symptoms than men. The proportion of adults affected by musculoskeletal diseases increased strongly with age.

Conclusion. Musculoskeletal disorders and symptoms occur frequently. The burden of complaints and diagnoses is comparable to previous population-based surveys.

\section{Keywords}

Musculoskeletal disorders - Back pain . Osteoarthritis - Arthritis - Osteoporosis . General population von 3 bzw. 4 Seiten verschiebbar war, oder bei einer proliferativen Schwellung über dem Gelenkspalt angenommen, die bei Druck elastisch und verschiebbar war. Nicht als Schwellung zählte eine derbe, „knochenharte“, nicht verschiebbare Auftreibung des Knochens. Die Teilnehmenden wurden zuvor aufgefor- dert, empfundene Schmerzen bei der Untersuchung zu äußern. Jede Gelenkuntersuchung endete mit der gezielten Frage, ob der Druck auf das Gelenk schmerzt. Als Druckschmerz wurde jede Form von Schmerzreaktion gewertet und diese für jedes Gelenk dokumentiert. Beispiele für Schmerzreaktionen waren: spontane Schmerzäußerung, Angabe von Schmerz auf Befragen oder Fluchtreaktionen.

Bei der Winkelstuhluntersuchung wurde, nachdem die teilnehmende Person auf dem Winkelstuhl Platz genommen hatte, zunächst das Vorliegen von Ruheschmerzen an Knie und Hüftgelenk 
Tab. 1 Analysierte Interviewfragen mit muskuloskelettalem Bezug der NAKO Gesundheitsstudie

\section{Sektion: muskuloskelettale Erkrankungen}

\section{Fragentext}

Wurde bei Ihnen jemals von einem Arzt eine der folgenden muskuloskelettalen Erkrankungen diagnostiziert?

Hatten Sie in den letzten 12 Monaten 3 Monate oder länger fast täglich Rückenschmerzen und wenn Ja, wie stark waren diese ausgeprägt?

Wurden Sie in den letzten 12 Monaten wegen einer Osteoporose ärztlich behandelt?

An welchem der folgenden Gelenke wurde bei Ihnen jemals von einem Arzt Arthrose bzw. Verschleiß diagnostiziert?

Wurden Sie in den letzten 12 Monaten wegen einer Arthrose der Hüftgelenke ärztlich behandelt?

Wurden Sie in den letzten 12 Monaten wegen einer Arthrose der Kniegelenke ärztlich behandelt?

Wurden Sie in den letzten 12 Monaten wegen einer Arthrose der Fingergelenke ärztlich behandelt?

\section{Sektion: seltene Erkrankungen}

Fragentext

Wurde bei Ihnen jemals von einem Arzt eine oder mehrere der folgenden seltenen Erkrankungen diagnostiziert?

Wurden Sie wegen einer der von Ihnen genannten Erkrankungen in den letzten 12 Monaten ärztlich behandelt?

\section{Antwortmöglichkeiten}

Rückenschmerzen über 3 Monate oder länger, und zwar fast täglich

Osteoporose

Arthrose bzw. Gelenkverschleiß

Nein, ich hatte keine andauernden Rückenschmerzen

Ja, gering

Ja, mäßig

Ja, stark

Ja

Nein

Hüftgelenke

Kniegelenke

Fingergelenke

Sonstiges Gelenk

Ja

Nein

Ja

Nein

Ja

Nein

\section{Antwortmöglichkeiten $^{\mathrm{a}}$}

Rheumatoide Arthritis/Polyarthritis

Morbus Bechterew/ankylosierende Spondylitis

Systemischer Lupus erythematodes

Sjögren-Syndrom

Rheumatoide Arthritis/Polyarthritis

Morbus Bechterew/ankylosierende Spondylitis

Systemischer Lupus erythematodes

Sjögren-Syndrom

${ }^{a}$ Bei den Antwortmöglichkeiten zu seltenen Erkrankungen sind nur die berücksichtigten Kategorien aufgeführt

erfragt. Dann erfolgte die Untersuchung der passiven Hüft- und Kniebeweglichkeit als Hinweis auf eine mögliche Bewegungseinschränkung sowie auf knöcherne Anbauten oder Bewegungsreiben bei aktiver Bewegung am Kniegelenk. Ergebnisse zu letzteren Untersuchungen wurden in der vorliegenden Arbeit nicht berücksichtigt, da der Fokus dieses Papers auf muskuloskelettalen Diagnosen und Schmerzen bzw. Schwellungen liegt.
Den Untersuchungen liegen standardisierte Prozeduren (SOP) zugrunde, die Untersucher wurden geschult und an Gesunden und Patienten trainiert. Hierzu wurden sowohl zentrale Schulungen als auch Einweisungen am jeweiligen Zentrum durchgeführt. Mittels Intraklassenkorrelation berechnete Untersuchereinflüsse liegen für die einzelnen Items zu Schmerzen $\leq 0,01$ sowie für Schwellungen $\leq 0,04$, wobei ein niedrigerer Wert einen geringeren Untersuchereinfluss indiziert.

\section{Auswertung}

Die Beschreibung der Verteilung einzelner Variablen erfolgte mittels absoluter $(N)$ und relativer Häufigkeiten (\%). Wegen der stratifizierten Stichprobenziehung mit Untergewichtung der Studienteilnehmenden unter 40 Jahren wurden die Ergebnisse nach Geschlecht und Altersgruppe in 5-Jahres-Schritten auf die deutsche Standardbevölkerung 2011 standardisiert [27]. Die ungewichteten Ergebnisse sind im Online-Zusatzmaterial (Tab. S1, S2 und S3) zu finden; bei ihnen fallen die relativen Häufigkeiten entsprechend dem geringeren Ziehungsanteil der unter 40-Jährigen für die untersuchten Erkrankungen konsistent höher aus.

Die Assoziationen der Outcomes mit dem Alter wurden mittels fraktionaler Polynome modelliert, um potenziell nichtlineare Zusammenhänge besser $\mathrm{zu}$ beschreiben [28]. Zugelassen wurden für den Haupteffekt des Alters funktionale Formen mit bis zu 3 Freiheitsgraden, Potenzen wurden aus der Menge [-2 $\left.\begin{array}{lll}-1(.5) & 2 & 3\end{array}\right]$ ausgewählt. Ein Test zwischen unterschiedlichen funktionalen Formen fand auf Basis von Devianztests mit $p<0,01$ statt. Die binären Endpunkte wurden mittels logistischer Regression modelliert, als Ergebnis sind in den Grafiken vorhergesagte Wahrscheinlichkeiten angegeben. Prädiktoren waren Alter in Jahren, Geschlecht sowie ein Produktterm zwischen Alter (zentriert) und Geschlecht.

Für die Hand- und Winkelstuhluntersuchung wurden mehrere Ausschlusskriterien definiert. Eingeschlossen wurden nur Ergebnisse von Studienassistenten, die mindestens 30 Personen untersucht haben, um einen ausreichenden Trainingsstand sicherzustellen. Die Winkelstuhluntersuchung wurde von insgesamt 119 Untersuchern durchgeführt, davon untersuchten 56 mindestens 30 Teilnehmende. Nach Ausschluss der Studienassistenten mit wenigen Untersuchungen verblieben 8470 (94,9\%) Teilnehmende für die weiteren Auswertungen. Bei der Handuntersuchung verblieben 57 von 122 Studienassistenten mit insgesamt 9155 (94,6\%) untersuchten Teilnehmenden. Zudem wurde ein Untersucher 
Tab. 2 Relative Häufigkeiten muskuloskelettaler Erkrankungen, alters- und geschlechtsstandardisiert, in der NAKO Gesundheitsstudie zur Halbzeit der Basiserhebung

\begin{tabular}{|c|c|c|c|c|}
\hline & & $\begin{array}{l}\text { Gesamt } \\
\text { (\%) }\end{array}$ & $\begin{array}{l}\text { Männer } \\
\text { (\%) }\end{array}$ & $\begin{array}{l}\text { Frauen } \\
\text { (\%) }\end{array}$ \\
\hline \multicolumn{5}{|l|}{ Rückenschmerz } \\
\hline \multicolumn{2}{|c|}{ Jemals Rückenschmerz für 3 und mehr Monate } & 22,48 & 19,69 & 25,23 \\
\hline \multirow{3}{*}{$\begin{array}{l}\text { Rückenschmerzen } \\
\geq 3 \text { Monate in den letzten } \\
12 \text { Monaten }\end{array}$} & Gering & 3,15 & 3,07 & 3,24 \\
\hline & Mäßig & 7,03 & 5,55 & 8,48 \\
\hline & Stark & 3,87 & 2,89 & 4,84 \\
\hline \multicolumn{5}{|l|}{ Osteoporose } \\
\hline \multicolumn{2}{|c|}{ Osteoporose jemals diagnostizierte } & 2,89 & 1,38 & 4,39 \\
\hline \multicolumn{2}{|c|}{ Osteoporose in den letzten 12 Monaten behandelt } & 1,28 & 0,54 & 2,02 \\
\hline \multicolumn{5}{|l|}{ Arthrose } \\
\hline \multicolumn{2}{|l|}{ Jemals Arthrose } & 20,55 & 17,42 & 23,64 \\
\hline \multicolumn{2}{|c|}{ Jemals Arthrose der Hüftgelenke } & 4,58 & 3,97 & 5,19 \\
\hline \multicolumn{2}{|c|}{ Jemals Arthrose der Hüftgelenke ärztlich behandelt } & 1,69 & 1,25 & 2,12 \\
\hline \multicolumn{2}{|c|}{ Jemals Arthrose der Kniegelenke } & 10,64 & 9,43 & 11,83 \\
\hline \multicolumn{2}{|c|}{ Jemals Arthrose der Kniegelenke ärztlich behandelt } & 3,53 & 2,83 & 4,22 \\
\hline \multicolumn{2}{|c|}{ Jemals Arthrose der Fingergelenke } & 5,88 & 2,68 & 9,04 \\
\hline \multicolumn{2}{|c|}{ Jemals Arthrose der Fingergelenke ärztlich behandelt } & 1,56 & 0,72 & 2,39 \\
\hline \multicolumn{2}{|c|}{ Jemals Arthrose der sonstige Gelenke } & 9,81 & 8,13 & 11,47 \\
\hline \multicolumn{5}{|c|}{ Arthritiden } \\
\hline \multicolumn{2}{|l|}{ Rheumatoide Arthritis } & 1,85 & 1,08 & 2,62 \\
\hline \multicolumn{2}{|c|}{ Morbus Bechterew/ankylosierende Spondylitis } & 0,49 & 0,55 & 0,42 \\
\hline \multicolumn{2}{|c|}{ Systemischer Lupus erythematodes } & 0,14 & 0,05 & 0,23 \\
\hline \multicolumn{2}{|l|}{ Sjögren-Syndrom } & 0,07 & 0,01 & 0,13 \\
\hline \multicolumn{5}{|c|}{$\begin{array}{l}\text { Dargestellt sind auf die deutsche Standardbevölkerung } 2011 \text { gewichtete Prozente. Den standardi- } \\
\text { sierten Prozentwerten liegen folgende Stichprobengrößen zugrunde: gesamt: } N=101.779 ; \text { Männer: } \\
N=47.259 ; \text { Frauen: } N=54.520 \\
\text { Die Angaben zu den Häufigkeiten der Erkrankungen beziehen sich auf jemals ärztlich diagnostizierte } \\
\text { Erkrankungen. Die Angaben zur ärztlichen Behandlung beziehen sich auf die letzten } 12 \text { Monate } \\
\text { vor der Erhebung. Der Anteil fehlender Werte je Item liegt jeweils }<1 \% \text { mit einer Anzahl zwischen } \\
n=311 \text { und } n=895\end{array}$} \\
\hline
\end{tabular}

Tab. 3 Relative Häufigkeiten, alters- und geschlechtsstandardisiert, von Schmerzen und Schwellungen in den Fingergelenken. Daten der NAKO Gesundheitsstudie zur Halbzeit der Basiserhebung

\begin{tabular}{|c|c|c|c|}
\hline & Gesamt (\%) & Männer (\%) & Frauen (\%) \\
\hline $\begin{array}{l}\text { Schmerzen mindestens ein Fingergelenk an einer } \\
\text { Hand }\end{array}$ & 5,99 & 3,79 & 8,50 \\
\hline $\begin{array}{l}\text { Schmerzen mindestens ein Fingergelenk linke } \\
\text { Hand }\end{array}$ & 4,03 & 2,43 & 5,87 \\
\hline $\begin{array}{l}\text { Schmerzen mindestens ein Fingergelenk rechte } \\
\text { Hand }\end{array}$ & 4,23 & 2,34 & 6,39 \\
\hline$\geq 2$ Schwellungen an den Fingergelenken & 2,40 & 1,46 & 3,48 \\
\hline $\begin{array}{l}\geq 2 \text { Schwellungen an den Fingergelenken linke } \\
\text { Hand }\end{array}$ & 1,74 & 1,02 & 2,57 \\
\hline $\begin{array}{l}\geq 2 \text { Schwellungen an den Fingergelenken rechte } \\
\text { Hand }\end{array}$ & 2,01 & 1,26 & 2,87 \\
\hline \multicolumn{4}{|c|}{$\begin{array}{l}\text { Dargestellt sind auf die deutsche Standardbevölkerung im Jahr } 2011 \text { gewichtete Prozente } \\
\text { Den standardisierten Prozentwerten liegen folgende Stichprobengrößen zugrunde: Handuntersu- } \\
\text { chung: gesamt: } N=9076 \text {; Männer: } N=4480 \text {; Frauen: } N=4596 \\
\text { Der Anteil fehlender Werte je Item liegt }<0,2 \%\end{array}$} \\
\hline
\end{tabular}

ausgeschlossen, bei dem im Rahmen der Qualitätssicherung mutmaßliche Falscheintragungen auffielen, begründet durch Serien von alternierenden, inhaltlich unmöglichen Ja-/Nein-Eintragungen. Sämtliche Datenwerte dieses Untersuchers $(n=122)$ wurden nicht berücksichtigt. Überdies wurden bei der Winkelstuhluntersuchung 80 Teilnehmende mit einem Körpergewicht über $130 \mathrm{~kg}$ ausgeschlossen, die entgegen der SOP-Anweisung untersucht wurden, obwohl der Winkelstuhl nicht für ein solches Übergewicht konzipiert ist. Bei 83 Teilnehmenden konnte keine vollständige Knie-, bei 95 Teilnehmenden keine Hüftuntersuchung stattfinden, was vor allem bei künstlichem Gelenkersatz der Fall war. Da sowohl die Knie- als auch die Hüftuntersuchung im Winkelstuhl stattfanden, wurden alle Teilnehmenden ausgeschlossen, bei denen mindestens ein Gelenk nicht untersucht werden konnte. Damit verblieben für die Analysen zum Winkelstuhl 8051 (90,2\%) der 8928 Teilnehmenden, die das Modul begonnen hatten. Für die Handuntersuchung betrug die entsprechende Zahl 9076 (93,3\%) von 9725 Teilnehmenden.

Alle Analysen wurden syntaxbasiert durchgeführt mit Stata 15 (Version 15.1, Stata Inc ${ }^{\circledR}$, College Station, TX, USA).

\section{Ergebnisse}

\section{Stichprobenbeschreibung und Teilnahme an den Untersuchungen}

Im Beobachtungszeitraum nahmen in den 18 bundesweit verteilten Studienzentren 54.520 Frauen (53,6\%; Alter Mittelwert (M): 51,5 Jahre, Standardabweichung (SD): 12,35; Spannbreite 20-73 Jahre) und 47.259 Männer (46,4\%; Alter M: 52,5 Jahre, SD: 12,32; Spannbreite 20-75 Jahre) am Interview (Level 1) der NAKO Gesundheitsstudie teil.

Dieklinischen Untersuchungen an der Hand sowie an den Knien und Hüften wurden an insgesamt 11 Studienzentren angeboten. Von den 9370 Teilnehmenden, die nach den o.g. Ausschlüssen berücksichtigt wurden $(n=7757$ nahmen an beiden Untersuchungen teil, 294 nur an der Winkelstuhluntersuchung, 1319 
Schwellung des Gelenkes

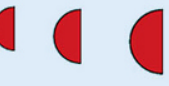

$0,5 \% \quad 0,75 \% \quad 1,0 \% \quad 1,25 \% \quad 1,5 \% \quad 1,75 \%$

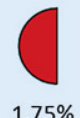

Schmerzen des Gelenkes ( $n=8.072$ )
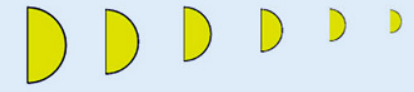

$\begin{array}{lllllll}1,75 \% & 1,5 \% & 1,25 \% & 1,0 \% & 0,75 \% & 0,5 \%\end{array}$
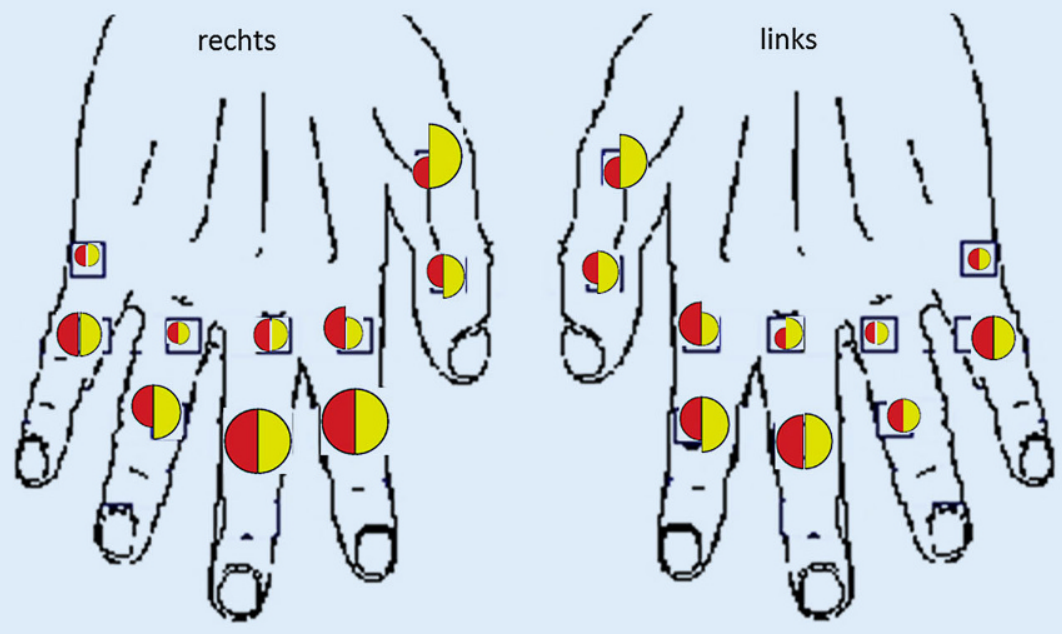

Abb. $1 \Delta$ Verteilung von Schmerzen und Schwellungen in den Fingergelenken aufBasis der klinischen Handuntersuchung. Dargestellt sind auf die deutsche Standardbevölkerung im Jahr 2011 gewichtete Wahrscheinlichkeiten. Daten der NAKO Gesundheitsstudie zur Halbzeit der Basiserhebung

nur an der Handuntersuchung), waren 4746 Frauen (50,4\%; Alter M: 50,9 Jahre, SD: 11,9; Spannbreite 20-73 Jahre) und 4624 Männer (49,6\%; Alter M: 51,4 Jahre, SD: 12,0; Spannbreite 20-72 Jahre).

\section{Häufigkeit muskuloskelettaler Erkrankungen im Überblick}

Auf der Grundlage der Selbstberichte der Teilnehmenden sind die jemals diagnostizierten andauernden Rückenschmerzen $(22,5 \%)$ sowie Erkrankungen aus dem Bereich der Arthrosen (20,6\%) am häufigsten (• Tab. 2). Mit jeweils ca. $11 \%$ folgten die Arthrose der Kniegelenke (Gonarthrose) sowie die mäßigen bis starken Rückenschmerzen. Die Osteoporose trat seltener auf (2,9\%).

Unter den jemals diagnostizierten Erkrankungen aus dem Formenkreis rheumatischer Erkrankungen wurde die rheumatoide Arthritis mit 1,9\% am häufigsten genannt. Ein jemals diagnostizierter Morbus Bechterew, systemischer Lupus erythematodes oder ein Sjögren-Syndrom wurde jeweils von deutlich weniger als $1 \%$ der Teilnehmenden berichtet.

Die Handuntersuchung ergab bei 6,0\% der Teilnehmenden Schmerzen an mindestens einem Fingergelenk sowie bei 2,4\% Schwellungen an mindestens zwei Gelenken (- Tab. 3). Die Fingermittelgelenke waren konsistent häufiger von Schmerzen bzw. Schwellungen betroffen als die Fingergrundgelenke ( $\bullet$ Abb. 1). Die rechte Hand war stärker betroffen als die linke, vor allem am Zeige- und Mittelfinger.

In der Winkelstuhluntersuchung lag bei 8,2 \% ein Ruheschmerz in mindestens einem Knie vor (• Tab. 4). Mindestens eine Hüfte war bei 5,1\% von Ruheschmerzen betroffen.

Frauen berichteten häufiger von muskuloskelettalen Erkrankungen als Männer mit Ausnahme von Morbus Bechterew, den Männer geringfügig häufiger angaben ( Tab. 2). Die Häufigkeiten nahmen mit dem Alter zu (• Abb. 2). Je nach Erkrankung bzw. Symptom fielen die Effekte von Alter und Geschlecht unterschiedlich stark aus. Hinsichtlich der Geschlechterunterschiede hoben sich die Osteoporose und die meisten entzündlichen Gelenkerkrankungen von

\section{Geschlechterverteilung und Alterswendigkeit}

den Rückenschmerzen sowie den Arthrosen durch ein im Verhältnis deutlich häufigeres Vorkommen bei Frauen ab. Der Zusammenhang des Alters mit der Häufigkeit von Rückenschmerzen unterschied sich charakteristisch von dem der anderen Erkrankungen: Der stärkste Anstieg trat bei Rückenschmerzen im jüngeren Erwachsenenalter auf, während dies bei den anderen untersuchten Erkrankungen erst im fortgeschrittenen Erwachsenenalter ab der 5. Lebensdekade der Fall war. Das Ausgangsniveau der selbst berichteten diagnostizierten muskuloskelettalen Erkrankungen lag bei 20 -Jährigen nahe bei $0 \%$, mit Ausnahme andauernder Rückenschmerzen, bei denen das Ausgangsniveau bei rund $6 \%$ lag.

\section{Diskussion}

Die vorliegenden Ergebnisse legen eine hohe Beschwerdelast durch muskuloskelettale Erkrankungen und Symptome in der erwachsenen Allgemeinbevölkerung nahe, insbesondere durch andauernde Rückenschmerzen und Arthrose. Die Beschwerdelast ist $\mathrm{zu}$ früheren bevölkerungsbezogenen Erhebungen überwiegend vergleichbar. Die berichteten Häufigkeiten können nicht als genaue Prävalenzschätzungen interpretiert werden, da dies weitere Analysen zu möglichem Selektionsbias durch das Sampling sowie zum Informationsbias erfordern würde.

Für die in der NAKO Gesundheitsstudie gewählte Operationalisierung zur Erfassung chronischer Rückenschmerzen ist der telefonische Gesundheitssurvey des Robert Koch-Instituts von 2003 ein wichtiger Bezugspunkt [9]. Die Angaben in unserer Studie, jemals chronische Rückenschmerzen gehabt $\mathrm{zu}$ haben, liegen zwar etwas höher als im Telefonsurvey, bei dem 15,5\% der Männer und $21,6 \%$ der Frauen chronische Rückenschmerzen angaben (NAKO: 19,7\% bzw. $25,2 \%$ ). Allerdings ist die Operationalisierung nicht identisch, was neben Stichprobenunterschieden zum beobachteten Unterschied beitragen kann. Gut vergleichbar mit Vorarbeiten ist, mit etwas über $10 \%$, der Anteil der Teilnehmenden, die mäßige bis starke 
Tab. 4 Relative Häufigkeiten, alters- und geschlechtsstandardisiert, von Ruheschmerz in Knieund Hüftgelenken aufBasis der Winkelstuhluntersuchung. Daten der NAKO Gesundheitsstudie zur Halbzeit der Basiserhebung

\begin{tabular}{|c|c|c|c|}
\hline & Gesamt (\%) & Männer (\%) & Frauen (\%) \\
\hline \multicolumn{4}{|c|}{ Anteil der Teilnehmer mit Ruheschmerzen im Knie } \\
\hline Nur linkes Knie & 5,24 & 4,73 & 5,82 \\
\hline Nur rechtes Knie & 5,84 & 5,28 & 6,49 \\
\hline Nur ein Knie & 5,41 & 5,33 & 5,48 \\
\hline Mindestens ein Knie & 8,24 & 7,74 & 8,82 \\
\hline Beide Knie & 2,83 & 2,26 & 3,49 \\
\hline \multicolumn{4}{|c|}{ Anteil der Teilnehmer mit Ruheschmerz der Hüften } \\
\hline Nur linke Hüfte & 3,23 & 2,52 & 4,04 \\
\hline Nur rechte Hüfte & 3,43 & 2,56 & 4,44 \\
\hline Nur eine Hüfte & 3,53 & 2,86 & 4,29 \\
\hline Mindestens eine Hüfte & 5,10 & 3,97 & 6,39 \\
\hline Beide Hüften & 1,57 & 1,11 & 2,10 \\
\hline \multicolumn{4}{|c|}{$\begin{array}{l}\text { Dargestellt sind auf die deutsche Standardbevölkerung } 2011 \text { gewichtete Prozente } \\
\text { Den standardisierten Prozentwerten liegen folgende Stichprobengrößen zugrunde: Winkelstuhlun } \\
\text { tersuchung: gesamt: } N=8051 \text {; Männer: } N=3992 ; \text { Frauen: } N=4059 \\
\text { Der Anteil fehlender Werte je Item liegt bei rund 2,75\% }\end{array}$} \\
\hline
\end{tabular}

Rückenschmerzen berichteten [29]. Die verbreitete Vermutung, dass Rückenschmerzen in der Bevölkerung ständig zunehmen, ist in den Jahren 1990 und 2003 mit Surveys aus Lübeck widerlegt worden [30]. Die Krankheitswertigkeit der berichteten Angaben zu Rückenschmerzen kann mit den vorliegenden Daten nur eingeschränkt bewertet werden. Dafür wären ergänzende Angaben zu Funktionsbeeinträchtigungen durch Rückenschmerzen notwendig gewesen.

Die Häufigkeit der behandelten Osteoporose steht im Einklang mit der GEDA-Gesundheitsstudie von 20132014, bei der die Daten ähnlich erhoben wurden [14]. Sie zeigen dieselben bekannten Zusammenhänge mit dem Alter sowie eine höhere Prävalenz bei Frauen. Laut Abrechnungsdaten erhalten ca. $2 \%$ der Versicherten ab 50 Jahren eine Therapie wegen Osteoporose [22]. Für jegliche Datenquellen, die auf Basis von Selbstberichten oder Versorgungsdaten basieren, ist eine erhebliche Unterschätzung der Prävalenz wahrscheinlich, da eine Osteoporose lange Zeit symptomfrei verläuft. Selbst bei einer für Osteoporose typischen Fraktur wird die Diagnose häufig nicht gestellt und es wird vermutet, dass nur $20 \%$ der Menschen mit Osteoporose behandelt werden [22].

Rund ein Fünftel der Teilnehmenden gab eine jemals diagnostizierte Arthrose an. Damit ist die Häufigkeit vergleichbar mit Daten der GEDA-2012-Studie, bei der $28 \%$ der Frauen und $20 \%$ der Männer eine entsprechende Angabe machten (NAKO: 17,4\% bzw. 23,6\%). Gleichzeitig liegen die Werte leicht unterhalb einer Bevölkerungsstudie in Herne, im Rahmen derer insgesamt 27,4\% eine Arthrose im Rahmen von Selbstangaben berichteten [31]. Es bestehen jedoch Unterschiede zwischen der anamnestischen Angabe diagnostizierter Arthrose sowie aktuell vorliegender Gelenkschmerzen in Interviews und der Dokumentation von Ruhe- bzw. Bewegungsschmerzen im Rahmen klinischer Untersuchungen. So ergaben Fragen in europäischen Surveys nach dem Vorliegen einer Hüftarthrose überwiegend eine geringere Häufigkeit $(0,9-7,0 \%$; [32-37]) als die Frage nach aktuell vorliegenden Hüftschmerzen (7,4-28,5\%; [33, 36, 38-40]). Umgekehrt ergab sich in der Framingham-Studie bei 19,6\% der untersuchten Teilnehmenden eine radiologisch gesicherte Arthrose (KellgrenLawrence Score $\geq 2$ ), obwohl nur 4,2\% von schmerzhaften Symptomen berichteten [12]. In unserer Kohorte liegen die Häufigkeiten bezüglich selbst berichteter Hüft- und Kniegelenksarthrose im Interview sowie von den entsprechenden Ruheschmerzen bei der Winkelstuhluntersuchung auf einem ähnlichen Niveau.
Vermutlich wird damit die tatsächliche Häufigkeit von Arthrose unterschätzt. Zukünftigen Auswertungen von Daten der NAKO Gesundheitsstudie wird unter Berücksichtigung der Magnetresonanztomographie(MRT)-Untersuchung eine hohe Bedeutung dabei zukommen, um die Ergebnisse der Selbstangaben und der klinischen Untersuchung besser einordnen zu können.

Die in dieser Studie ermittelte Häufigkeit der rheumatoiden Arthritis ist mit $1,9 \%$ etwas niedriger als in DEGS1 (2008-2009), in der 2,5\% der 18- bis 79Jährigen von einer ärztlich diagnostizierten rheumatoiden Arthritis berichteten [41]. Diese Quellen, die sich ausschließlich auf die selbst berichtete Diagnose beziehen, zeigen höhere Prävalenzen als eine regionale Befragung aus dem Jahr 1989 in Hannover, die 0,6-0,9\% ergab [42], sowie Abrechnungsdiagnosen, die im Bereich von 0,8-1,4\% lagen [25]. Ein möglicher Grund sind Unklarheiten hinsichtlich der Abgrenzung der Arthritis von der weitaus häufiger vorkommenden Arthrose. Eine höhere Prävalenz von über $4 \%$ für Deutschland schätzt eine andere Studie [43] zur globalen Krankheitslast der rheumatoiden Arthritis. Dort wurden aber mit den ICD10-Codes M05-M06.9 und M08.0-M08.89 noch weitere Erkrankungen miterfasst, wie die in Deutschland häufig in Abrechnungen klassifizierte undifferenzierte Polyarthritis (M06.9) und die juvenilen Arthritiden (M08).

Die dokumentierte Prävalenz des systemischen Lupus erythematodes ist mit $0,14 \%$ höher und die des Sjögren-Syndroms mit $0,07 \%$ niedriger als aufgrund bisheriger Daten erwartet, während die Geschlechterverteilung mit einer sehr deutlichen weiblichen Dominanz den Versorgungsdaten entspricht [24]. Die Häufigkeitsangaben zum systemischen Lupus erythematodes entsprechen eher Abrechnungsdaten von 2012 aus Großbritannien [44] als deutschen Abrechnungsdaten aus dem Jahre 2002 [45]. Hier könnten die häufigeren rein kutanen Verläufe zu einer Zunahme der Berichthäufigkeit „Lupus“ führen. Da nicht nach primären und sekundären Formen des Sjögren-Syndroms gefragt wurde, ist die Einordnung schwierig, 


\section{Leitthema}
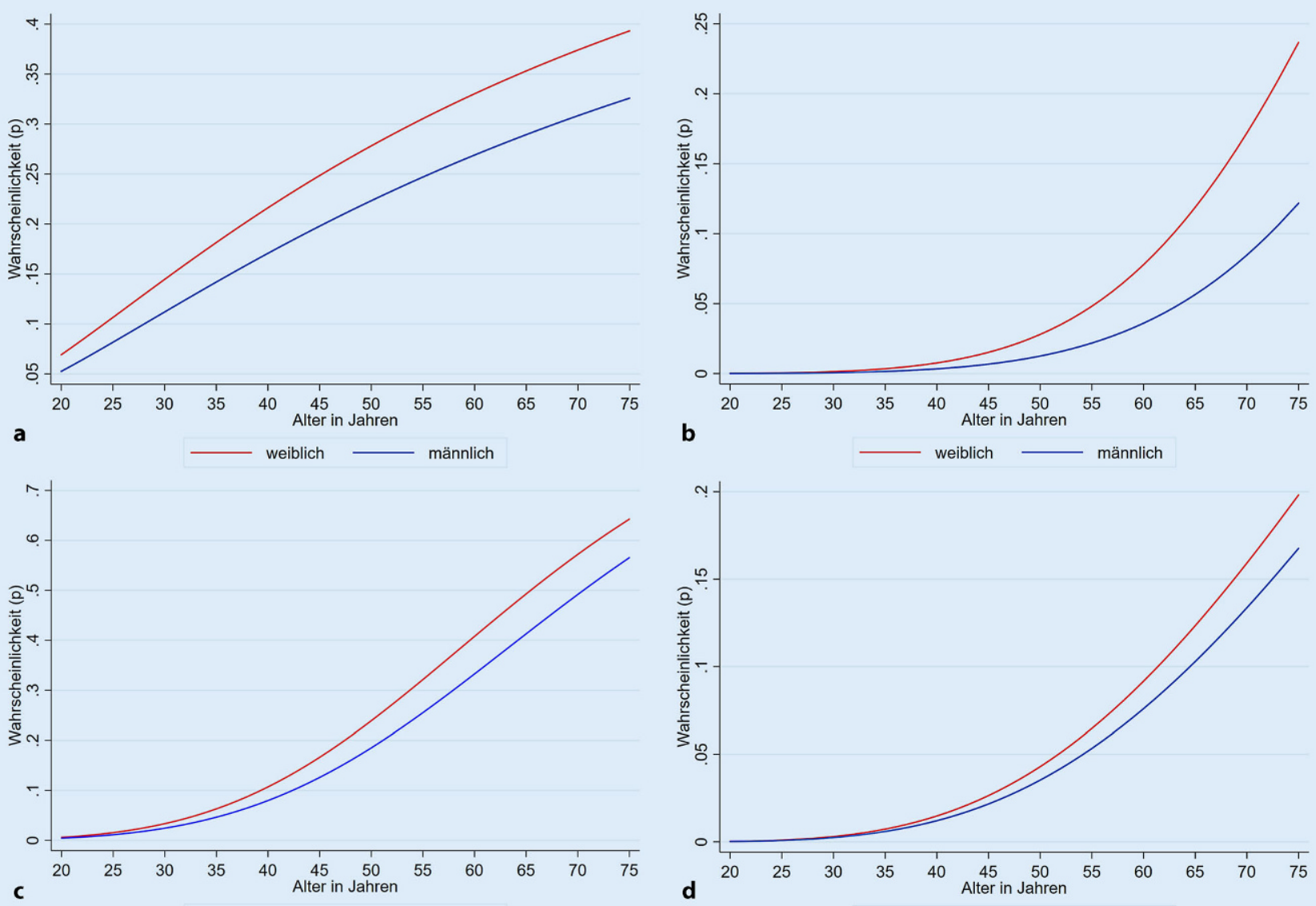

weiblich $\longrightarrow$ männlich
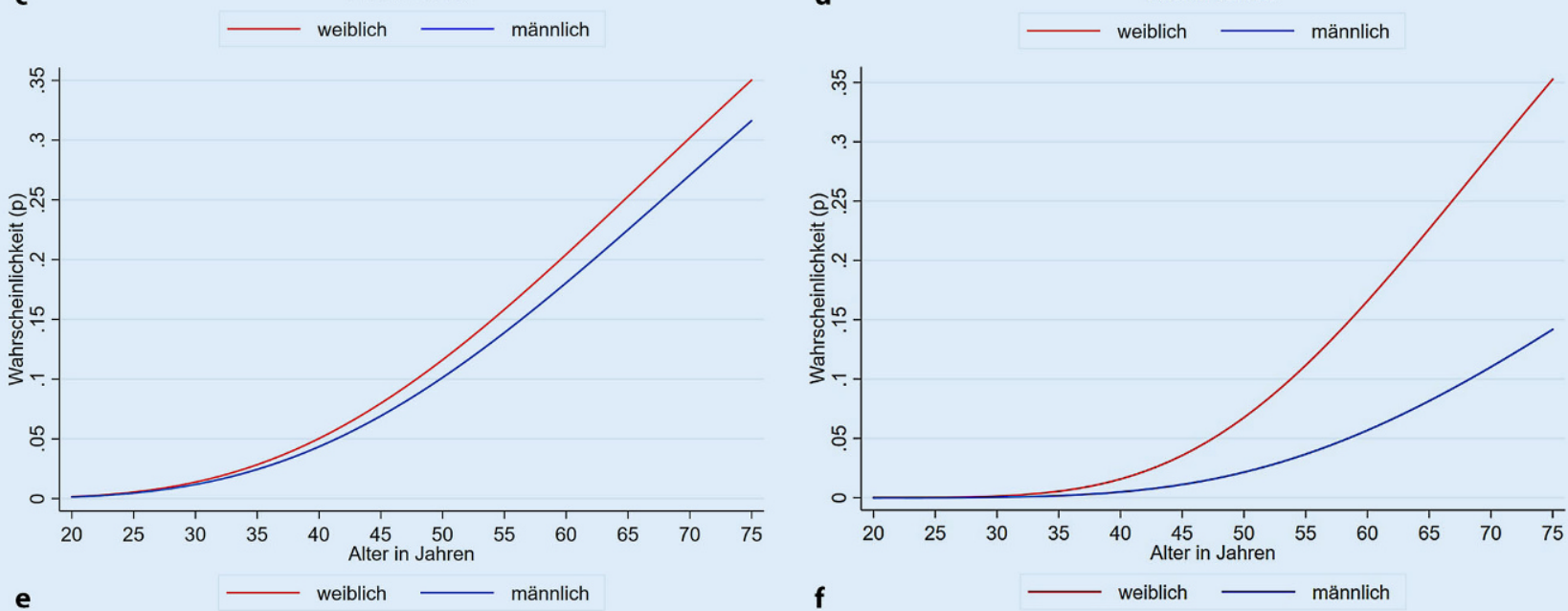

Abb. 2 A Altersverläufe jemals diagnostizierter muskuloskelettaler Symptome oderErkrankungen (a-h) aufBasis von Selbstangaben aus dem Interview. Daten der NAKO Gesundheitsstudie zur Halbzeit der Basiserhebung. Die dargestellten Wahrscheinlichkeiten basieren auf logistischen Regressionen mit Alter und Geschlecht sowie dem Produktterm zwischen Alter und Geschlecht. Das Alter wurde mittels Fractional Polynomials nichtlinearmodelliert. a Andauernde Rückenschmerzen, b Osteoporose, c Arthrose, $\mathbf{d}$ Arthrose der Hüftgelenke, $\mathbf{e}$ Arthrose der Kniegelenke, $\mathbf{f}$ Arthrose der Fingergelenke, $\mathbf{g}$ rheumatoide Arthritis, $\mathrm{h}$ Morbus Bechterew 

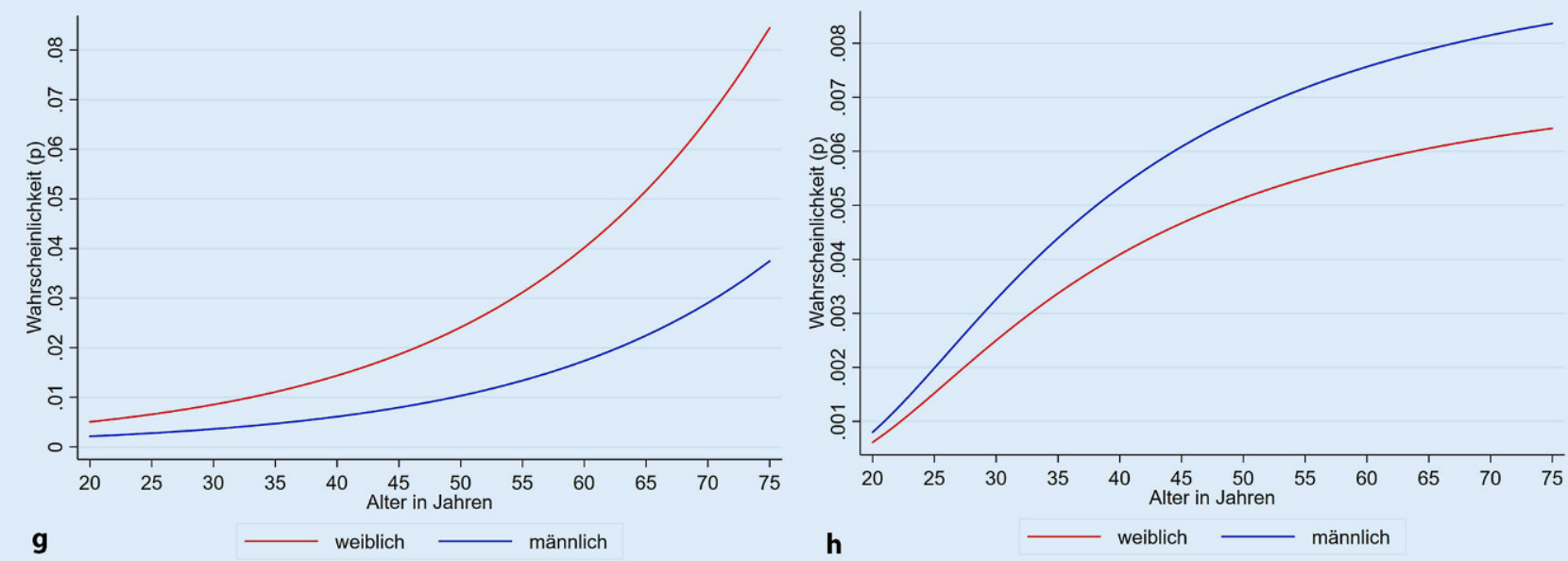

Abb. 2 × (Fortsetzung)

die Prävalenzangaben variieren je nach Falldefinition stark [46].

Bisherige publizierte klinische Untersuchungen beschränken sich oft auf sehr kleine Fallzahlen, obwohl unlängst die prognostische Überlegenheit der klinischen Untersuchung im Vergleich zur Befragung bei der Fingerpolyarthrose dargestellt wurde. Die vorliegende Studie ermittelt im Gegensatz dazu epidemiologisch erstmals mittels einer klinischen Untersuchung die Häufigkeitsverteilung von Schmerzen und Schwellungen in den Fingergelenken. Die Ergebnisse können zu einer weiteren Validierung der berichteten Diagnosen dienen. In DEGS1 wurden Fingergelenkschmerzen von $8 \%$ der Frauen und von 4\% der Männer angegeben [47]. Die Prozentwerte sind in der klinischen NAKO-Untersuchung vergleichbar (8,5\% bzw. 3,8\%). Die Verteilung der beobachteten Gelenkschwellungen auf die einzelnen Finger ähnelt den jeweils dokumentierten Schmerzen und legt einen Einfluss der natürlichen mechanischen Mehrbelastung der Daumen, Zeige- und Mittelfinger sowie der Mittelgelenke auf die Entstehung möglicher Symptome nahe.

\section{Stärken und Schwächen}

Stärken der Studie sind die große Stichprobengröße im Interview und die beiden klinischen Untersuchungen. Hinsichtlich Alter und Geschlecht ist die Auswahl der Teilnehmenden, die an der klinischen Untersuchung teilgenommen haben, der NAKO-Gesamtstichprobe ähnlich. Eine Schwäche ist die für Deutschland nichtrepräsentative Stichprobenziehung. Die NAKO Gesundheitsstudie wurde vor allem an Einrichtungen mit Vorerfahrungen in bevölkerungsbezogenen epidemiologischen Untersuchungen durchgeführt. Die berichteten Häufigkeiten können daher nicht als Prävalenzschätzungen für die deutsche Allgemeinbevölkerung interpretiert werden. Hinzu kommt, dass zwischen der Stichprobenziehung und der Untersuchung bis zu 6 Jahre vergehen konnten, was die Teilnahmewahrscheinlichkeit hoch morbider Personen gesenkt haben könnte. Ergänzende Analysen zu Selektionseffekten wären daher nötig, um die Generalisierbarkeit unserer Befunde auf die Allgemeinbevölkerung besser bewerten zu können. Zudem sollten alle Teilnehmenden der Basisstudie berücksichtigt werden. In diese Auswertung, die einen Zwischenstand darstellt, ging nur ca. die erste Hälfte der Stichprobe ein. Dieser Umstand schränkt die Vergleichbarkeit zu bisherigen Surveys ein, wenngleich die Ergebnisse insgesamt für eine ähnliche Beschwerdelast sprechen.

Informationsbias bei den retrospektiven Angaben hinsichtlich jemals diagnostizierter Erkrankungen ist wahrscheinlich. Es kann nicht davon ausgegangen werden, dass länger zurückliegende Ereignisse sicher erinnert werden [48]. Zudem muss von einem Problem bei der Abgrenzung von Arthrose und Arthritis ausgegangen werden: Ein Teil der Teilnehmenden, der von der weitaus häufiger auftretenden Arthrose betroffen ist, könnte diese fälschlich als Arthritis bezeichnet haben.

Bei der Durchführung der klinischen Untersuchung spielen potenzielle Untersuchereffekte eine Rolle. Vorliegende Analysen für Schmerzen legen einen eher geringen Einfluss nahe, bei Schwellungen ist der Einfluss etwas höher. Zudem haben viele Untersucher über lange Zeiträume jeweils nur sehr wenige Teilnehmende klinisch untersucht und sind deshalb nicht berücksichtigt worden. Dies hat die auswertbare Fallzahl aber nur geringfügig abgesenkt.

\section{Fazit}

Die Auswertungen der Daten zur Halbzeit der NAKO-Basiserhebung belegen eine große Verbreitung von muskuloskelettalen Erkrankungen und Symptomen, insbesondere von andauernden Rückenschmerzen und Arthrose. Die berechnete Beschwerdelast ist mit den Ergebnissen früherer bevölkerungsbezogener Erhebungen vergleichbar. Die berichteten Häufigkeiten können nicht als genaue Prävalenzschätzungen interpretiert werden. 


\section{Korrespondenzadresse}

Prof. Dr. Carsten Oliver Schmidt

Institut für Community Medicine - SHIP-KEF, Universitätsmedizin Greifswald

Walther-Rathenau-Str. 48, 17475 Greifswald, Deutschland

carsten.schmidt@uni-greifswald.de

Danksagung. Die Arbeitsgemeinschaft der Rheumazentren unterstützt die Untersuchungszentren auf regionaler Basis durch die Bereitstellung von Trainern und die fachliche Beratung. Unser Dank gilt allen Teilnehmerinnen und Teilnehmern sowie den Mitarbeiterinnen und Mitarbeitern der NAKO Gesundheitsstudie.

Förderung. Die NAKO Gesundheitsstudie wird durch das Bundesministerium für Bildung und Forschung (BMBF, Förderkennzeichen 01ER1301A/B/C und 01ER1511D), die Bundesländer und die Helmholtz-Gemeinschaft gefördert sowie durch die beteiligten Universitäten und Institute der LeibnizGemeinschaft finanziell unterstützt. Die Deutsche Gesellschaft für Rheumatologie (DGRh) und die Deutsche Gesellschaft für Orthopädie und Orthopädische Chirurgie (DGOOC) unterstützen die Schulungsmaßnahmen finanziell durch Reisekosten- und Aufwandsentschädigungen für das Schulungspersonal.

Funding. Open Access funding provided by Projekt DEAL.

\section{Einhaltung ethischer Richtlinien}

Interessenkonflikt. C.O. Schmidt, K.-P. Günther, J.Goronzy, K. Albrecht, J.-F. Chenot, J. Callhoff, A. Richter, R. Kasch, W. Ahrens, H. Becher, K. Berger, H. Brenner, B. Fischer, C.-W. Franzke, W. Hoffmann, B. Holleczek, L. Jaeschke, C. Jenning, K.-H. Jöckel, R. Kaaks, T. Keil, A. Kluttig, G. Krause, O. Kuß, M. Leitzmann, W. Lieb, J. Linseisen, M. Löffler, C. Meinke-Franze, C. Meisinger, K.B. Michels, R. Mikolajczyk, N. Obi, A. Peters, T. Pischon, T. Schikowski, S. Schipf, C. Specker, H. Völzke, K. Wirkner, A. Zink und O. Sander geben an, dass kein Interessenkonflikt besteht.

Das Studienprotokoll der NAKO wurde durch die zuständigen lokalen Ethikkommissionen und Datenschutzbeauftragten geprüft und positiv bewertet. Alle beschriebenen Untersuchungen stehen im Einklang mit nationalem Recht sowie der Deklaration von Helsinki in der aktuellen und überarbeiteten Fassung. Alle Teilnehmenden haben ihr Einverständnis zur Teilnahme an den Untersuchungen erklärt.

Open Access Dieser Artikel wird unter der Creative Commons Namensnennung 4.0 International Lizenz veröffentlicht, welche die Nutzung, Vervielfältigung, Bearbeitung, Verbreitung und Wiedergabe in jeglichem Medium und Format erlaubt, sofern Sie den/die ursprünglichen Autor(en) und die Quelle ordnungsgemäß nennen, einen Link zur Creative Commons Lizenz beifügen und angeben, ob Änderungen vorgenommen wurden.

Die in diesem Artikel enthaltenen Bilder und sonstiges Drittmaterial unterliegen ebenfalls der genannten
Creative Commons Lizenz, sofern sich aus der Abbildungslegende nichts anderes ergibt. Sofern das betreffende Material nicht unter der genannten Creative Commons Lizenz steht und die betreffende Handlung nicht nach gesetzlichen Vorschriften erlaubt ist, ist für die oben aufgeführten Weiterverwendungen des Materials die Einwilligung des jeweiligen Rechteinhabers einzuholen.

Weitere Details zur Lizenz entnehmen Sie bitte der Lizenzinformation auf http://creativecommons.org/ licenses/by/4.0/deed.de.

\section{Literatur}

1. von Renteln-Kruse W (2001) Epidemiologische Aspekte der Morbidität im Alter. Z Gerontol Geriat 34:I10-I15

2. Picavet H, Hoeymans N (2004) Health related quality of life in multiple musculoskeletal diseases: SF-36 and EQ-5D in the DMC3 study. Ann Rheum Dis 63:723-729

3. Maher C, Underwood M, Buchbinder R (2017) Nonspecific low back pain. Lancet 389:736-747

4. Sebbag E, Felten R, Sagez F, Sibilia J, Devilliers H, Arnaud $L$ (2019) The world-wide burden of musculoskeletal diseases: a systematic analysis of the world health organization burden of diseases database. Ann Rheum Dis 78:844-848

5. German National Cohort (GNC) Consortium (2014) The German national cohort: aims, study design and organization. Eur J Epidemiol 29:371-382

6. Hartvigsen J, Hancock MJ, Kongsted A et al (2018) What low back pain is and why we need to pay attention. Lancet 391:2356-2367

7. Hurwitz EL, Randhawa K, Torres P et al (2018) The global spine care initiative: a systematic review of individual and community-based burden of spinal disorders in rural populations in low- and middleincome communities. Eur Spine J 27:802-815

8. Schmidt CO, Raspe H, Pfingsten Met al (2007) Back pain in the German adult population: prevalence, severity, and sociodemographic correlates in a multiregional survey. Spine (Phila Pa 1976) 32:2005-2011

9. Neuhauser H, Ellert U, Ziese T (2005) Chronische Rückenschmerzen in der Allgemeinbevölkerung in Deutschland 2002/2003: Prävalenz und besonders betroffene Bevölkerungsgruppen. Gesundheitswesen 67:685-693

10. Chenot J-F, Haupt C, Gerste B (2014) Zeitliche Trends bei der Versorgung von Rückenschmerzpatienten. In: Klauber J, Günster C, Gerste B, Robra BP, Schmacke N (Hrsg) Versorgungs-Report 2013/2014. Schattauer, Stuttgart, S 155-184

11. Wenig CM, Schmidt CO, Kohlmann T, Schweikert B (2009) Costs of back pain in Germany. Eur J Pain 13:280-286

12. Kim C, Linsenmeyer KD, Vlad SC et al (2014) Prevalence of radiographic and symptomatic hip osteoarthritis in an urban United States community: the Framingham osteoarthritis study. Arthritis Rheumatol 66:3013-3017

13. Schneider S, Schmitt G, Mau H, Schmitt H, Sabo D Richter W (2005) Prevalence and correlates of osteoarthritis in Germany. Representative data from the first national health survey. Orthopade 34:782-790

14. Fuchs J, Kuhnert R, Scheidt-Nave C (2017) 12Monats-Prävalenz von Arthrose in Deutschland. JHealth Monit 2:55-60
15. Robert Koch-Institut (2014) Gesundheitsberichterstattung des Bundes, Faktenblatt zu GEDA 2012 Ergebnisse der Studie "Gesundheit in Deutschland aktuell 2012“

16. Statistisches Bundesamt (2019) Vollstationär behandelte Patientinnen und Patienten in Krankenhäuser 2018. https://www.destatis.de/ DE/Themen/Gesellschaft-Umwelt/Gesundheit/ Krankenhaeuser/Tabellen/drg-operationeninsgesamt.html\#fussnote-1-119748.Zugegriffen: 5.Dez. 2019

17. Fuchs J, Scheidt-Nave C, Kuhnert R (2017) 12-Monats-Prävalenz von Osteoporose in Deutschland. JHealth Monit 2:61-65

18. Scheidt-Nave C, Kamtsiuris P, Gosswald A et al (2012) German health interview and examination survey for adults (DEGS) — design, objectives and implementation of the first data collection wave. BMC Public Health 12:730

19. Holzer G (2013) Die Bedeutung der Osteoporose für den Orthopäden und Unfallchirurgen.ZOrthop Unfall 151:93-106

20. Dachverband Osteologie e. V. (2017) Prophylaxe Diagnostik und Therapie der Osteoporose. https:// www.awmf.org/leitlinien/detail/l//183-001.html. Zugegriffen:5.Dez. 2019

21. Compston JE, McClung MR, Leslie WD (2019) Osteoporosis. Lancet 393:364-376

22. Hadji P, Klein S, Gothe H et al (2013) The epidemiology of osteoporosis-bone evaluation study (BEST): an analysis of routine health insurance data. Dtsch Arztebl Int 110:52-57

23. SchneiderMP, Lelgemann M, AbholzHHetal (2011) Management der frühen rheumatoiden Arthritis. Springer, Berlin, Heidelberg, New York

24. Zink A, Albrecht K (2016) How frequent are musculoskeletal diseases in Germany? Z Rheumatol 75:346-353

25. Hense S, Ramos AL, Callhoff J, Albrecht K, Zink A, Hoffmann F (2016) Prävalenz der rheumatoiden Arthritis in Deutschland aufBasis von Kassendaten. ZRheumatol 75:819-827

26. German National Cohort Consortium (2015) The German national cohort-a prospective epidemiological study resource for health and disease research in Germany. https://nako.de/wpcontent/uploads/2015/07/WissenschaftlichesKonzept-der-NAKO2.pdf. Zugegriffen: 5. Dez. 2019

27. Robert Koch-Institut (2019) Informationssystem der Gesundheitsberichterstattung des Bundes. http://www.gbe-bund.de/ gbe10/abrechnung.prc_abr_test_logon?p uid=gast\&p_aid=43162945\&p_knoten=VR\& p_sprache $=D \& p \_s u c h s t r i n g=s t a n d a r d b e v$ \%F6lkerung.Zugegriffen: 5. Dez. 2019

28. Royston P, Sauerbrei W (2008) Multivariable model-building: a pragmatic approach to regression anaylsis based on fractional polynomials for modelling continuous variables. John Wiley \& Sons, Chichester

29. Schmidt CO, Raspe H, Kohlmann T (2010) Graded back pain revisited - do latent variable models change our understanding of severe back pain in the general population? Pain 149:50-56

30. Huppe A, Muller K, RaspeH (2007) Is the occurrence of back pain in Germany decreasing? Two regional postal surveys a decade apart. Eur J Public Health 17:318-322

31. Thiem U, Schumacher J, Zacher J, Burmester GR, Pientka L (2008) Prevalence of musculoskeletal complaints and self-reported joint osteoarthritis in the population of Herne : a telephone survey. ZRheumatol 67:432-439 
32. van Schaardenburg $D$, Van den Brande KJ, Ligthart GJ, Breedveld FC, Hazes JM (1994) Musculoskeletal disorders and disability in persons aged 85 and over: a community survey. Ann Rheum Dis 53:807-811

33. Skytta ET, Jarkko L, Antti E, Huhtala H, Ville R (2011) Increasing incidence of hip arthroplasty for primary osteoarthritis in 30- to 59-year-old patients. Acta Orthop 82:1-5

34. SalaffiF, De Angelis R, GrassiW (2005) Prevalence of musculoskeletal conditions in an Italian population sample: results of a regional community-based study. I. The MAPPING study. Clin Exp Rheumatol 23:819-828

35. Picavet HS, Schouten JS (2003) Musculoskeletal pain in the Netherlands: prevalences, consequences and risk groups, the DMC(3)-study. Pain 102:167-178

36. Grotle M, Hagen KB, Natvig B, Dahl FA, Kvien TK (2008) Prevalence and burden of osteoarthritis: results from a population survey in Norway. JRheumatol 35:677-684

37. Andrianakos AA, Kontelis LK, Karamitsos DG et al (2006) Prevalence of symptomatic knee, hand, and hip osteoarthritis in Greece. The ESORDIG study. JRheumatol 33:2507-2513

38. Reijman M, Hazes JM, Pols HA, Koes BW, BiermaZeinstra SM (2005) Acetabular dysplasia predicts incident osteoarthritis of the hip: the Rotterdam study. Arthritis Rheum 52:787-793

39. Frankel S, Eachus J, Pearson N et al (1999) Population requirement for primary hip-replacement surgery: a cross-sectional study. Lancet 353:1304-1309

40. Dawson J, Linsell L, Zondervan K et al (2004) Epidemiology of hip and knee pain and its impact on overall health status in older adults. Rheumatology (Oxford) 43:497-504

41. Fuchs J, Rabenberg M, Scheidt-Nave C (2013) Prevalence of selected musculoskeletal conditions in Germany: results of the German health interview and examination survey for adults (DEGS1). Bundesgesundheitsblatt Gesundheitsforschung Gesundheitsschutz 56:678-686

42. Wasmus A, Kindel P, Mattussek S, Raspe HH (1989) Activity and severity of rheumatoid arthritis in Hannover/FRG and in one regional referral center. Scand J Rheumatol Suppl 79:33-44

43. Safiri S, Kolahi AA, Hoy D et al (2019) Global, regional and national burden of rheumatoid arthritis 1990-2017: a systematic analysis of the global burden of disease study 2017. Ann Rheum Dis 78:1463-1471

44. Rees F, Doherty M, Grainge M, Davenport G, Lanyon P, Zhang W (2016) The incidence and prevalence of systemic lupus erythematosus in the UK, 1999-2012. Ann Rheum Dis 75:136-141

45. Brinks R, Fischer-Betz R, Sander O, Richter JG, Chehab G, Schneider M (2014) Age-specific prevalence of diagnosed systemic lupus erythematosus in Germany 2002 and projection to 2030. Lupus 23:1407-1411

46. Stefanski AL, Tomiak C, Pleyer U, Dietrich T, Burmester GR, Dorner T (2017) The diagnosis and treatment of Sjogren's syndrome. Dtsch Arztebl Int 114:354-361

47. Fuchs J, Prütz F (2017) Prävalenz von Gelenkschmerzen in Deutschland. J Health Monit 2:66-71

48. Coughlin SS (1990) Recall bias in epidemiologic studies. JClin Epidemiol 43:87-91

\section{Affiliations}

${ }^{1}$ Institut für Community Medicine - SHIP-KEF, Universitätsmedizin Greifswald, Greifswald, Deutschland

${ }^{2}$ Universitätsklinikum Dresden, Dresden, Deutschland

${ }^{3}$ Deutsches Rheuma-Forschungszentrum Berlin, Berlin, Deutschland

${ }^{5}$ Leibniz-Institut für Präventionsforschung und Epidemiologie - BIPS, Bremen, Deutschland

${ }^{6}$ Universitätsklinikum Hamburg-Eppendorf, Hamburg, Deutschland

${ }^{7}$ Westfälische Wilhelms-Universität Münster, Münster, Deutschland

${ }^{8}$ Deutsches Krebsforschungszentrum (DKFZ), Heidelberg, Deutschland

${ }^{9}$ Ministerium für Soziales Gesundheit Frauen und Familie - Saarland, Saarbrücken, Deutschland

${ }^{10}$ Universitätsklinikum Essen, Essen, Deutschland

${ }^{11}$ Institut für Sozialmedizin, Epidemiologie und Gesundheitsökonomie, Charité - Universitätsmedizin Berlin, Berlin, Deutschland

${ }^{12}$ Helmholtz-Zentrum für Infektionsforschung (HZI), Braunschweig, Deutschland

${ }^{13}$ Institut für Biometrie und Epidemiologie, Deutsches Diabetes-Zentrum (DDZ), Leibniz-Zentrum für Diabetes-Forschung an der Heinrich-Heine-Universität Düsseldorf, Düsseldorf, Deutschland

${ }^{14}$ Fakultät für Medizin, Universität Regensburg (UR), Regensburg, Deutschland

${ }^{15}$ Christian-Albrechts-Universität Kiel, Kiel, Deutschland

${ }^{16}$ Institut für Medizinische Informatik, Statistik, und Epidemiologie (IMISE), Universität Leipzig, Leipzig, Deutschland

${ }^{17}$ Lehrstuhl für Epidemiologie, am UNIKA-T, LMU München, Augsburg, Deutschland

${ }^{18}$ SFG Klinische Epidemiologie, Helmholtz Zentrum München, Neuherberg, Deutschland

${ }^{19}$ Forschergruppe Molekulare Epidemiologie, Max-Delbrück-Centrum für Molekulare Medizin in der Helmholtz-Gemeinschaft (MDC), Berlin, Deutschland

${ }^{20}$ Partnerstandort Berlin, Deutsches Zentrum für Herz-Kreislauf-Forschung (DZHK), Berlin, Deutschland

${ }^{21} \mathrm{MDC} /$ BIH Biobank, Max-Delbrück-Centrum für Molekulare Medizin in der Helmholtz-Gemeinschaft (MDC) und Berlin Institute of Health (BIH), Berlin, Deutschland

${ }^{22}$ Institut für Prävention und Tumorepidemiologie, Universitätsklinikum Freiburg, Medizinische Fakultät, Albert-Ludwigs-Universität Freiburg, Freiburg, Deutschland

${ }^{23}$ Martin-Luther-Universität Halle-Wittenberg, Halle (Saale), Deutschland

${ }^{24}$ IUF Leibniz-Institut für umweltmedizinische Forschung gGmbH, Düsseldorf, Deutschland

${ }^{25}$ Klinik für Rheumatologie \& Klinische Immunologie, Evang. Kliniken Essen-Mitte, Essen, Deutschland

${ }^{26}$ Leipziger Forschungszentrum für Zivilisationserkrankungen (LIFE), Universität Leipzig, Leipzig, Deutschland

${ }^{27}$ Klinik für Rheumatologie und Klinische Immunologie, Charité Universitätsmedizin Berlin, Berlin, Deutschland

${ }^{28}$ Poliklinik und Funktionsbereich für Rheumatologie, Heinrich-Heine-Universität, Düsseldorf, Deutschland

${ }^{29}$ Klinik und Poliklinik für Orthopädie und Chirurgische Orthopädie, Universitätsmedizin Greifswald, Greifswald, Deutschland

${ }^{30}$ Institut für Klinische Epidemiologie und Biometrie, Universität Würzburg, Würzburg, Deutschland

${ }^{31}$ Landesinstitut für Gesundheit, Bayerisches Landesamt für Gesundheit und Lebensmittelsicherheit, Bad Kissingen, Deutschland

${ }^{32}$ Charité - Universitätsmedizin Berlin, Berlin, Deutschland 